\title{
AdS Black Hole with Phantom Scalar Field
}

\author{
Limei Zhang, ${ }^{1}$ Xiaoxiong Zeng, ${ }^{2}$ and Zhonghua $\mathrm{Li}^{3}$ \\ ${ }^{1}$ College of Physics and Space Science, China West Normal University, Nanchong, Sichuan 637002, China \\ ${ }^{2}$ Institute of Theoretical Physics, Chinese Academy of Sciences, Beijing 100190, China \\ ${ }^{3}$ Institute of Theoretical Physics, China West Normal University, Nanchong, Sichuan 637002, China \\ Correspondence should be addressed to Zhonghua Li; sclzh888@163.com
}

Received 6 July 2017; Accepted 2 August 2017; Published 15 October 2017

Academic Editor: $\mathrm{Li} \mathrm{Li}$

Copyright ( 2017 Limei Zhang et al. This is an open access article distributed under the Creative Commons Attribution License, which permits unrestricted use, distribution, and reproduction in any medium, provided the original work is properly cited. The publication of this article was funded by $\mathrm{SCOAP}^{3}$.

\begin{abstract}
We present an AdS black hole solution with Ricci flat horizon in Einstein-phantom scalar theory. The phantom scalar fields just depend on the transverse coordinates $x$ and $y$, which are parameterized by the parameter $\alpha$. We study the thermodynamics of the AdS phantom black hole. Although its horizon is a Ricci flat Euclidean space, we find that the thermodynamical properties of the black hole solution are qualitatively the same as those of AdS Schwarzschild black hole. Namely, there exists a minimal temperature and the large black hole is thermodynamically stable, while the smaller one is unstable, so there is a so-called Hawking-Page phase transition between the large black hole and the thermal gas solution in the AdS space-time in Poincare coordinates. We also calculate the entanglement entropy for a strip geometry dual to the AdS phantom black holes and find that the behavior of the entanglement entropy is qualitatively the same as that of the black hole thermodynamical entropy.
\end{abstract}

\section{Introduction}

Due to its confined boundary of anti-de Sitter (AdS) spacetime, thermodynamics of black hole in AdS space and those in flat or de Sitter space-time are very different. For example, for a Schwarzschild AdS black hole, it is stable if its horizon is larger than a certain value, while, for a small black hole, the effect of cosmological constant can be neglected, the black hole behaves just like a Schwarzschild black hole in flat space, and it is thermodynamically unstable. In addition, it is found that there is an AdS black hole only when the temperature of black hole is larger than a critical value, while there is only a thermal gas solution when its temperature is less than the value. The first-order phase transition which occurs between the black hole solution and the thermal gas solution in the AdS space-time is named Hawking-Page phase transition [1]. The phase transition gets its interpretation following the AdS/CFT correspondence [2-5].

Another big difference between black holes in AdS space and flat or de Sitter space-time is that the horizon of black holes in flat or de Sitter space must have a spherical structure, while the horizon topology of black holes in AdS space-time could be a zero or negative constant curvature surface, except for the case with a positive constant curvature surface. These kinds of black holes with zero or negative constant curvature horizon have been studied in the literature [6-19]: they are usually called topological AdS black holes. Particularly, we find that these so-called topological AdS black holes are always thermodynamically stable, and there do not exist Hawking-Page phase transitions related to those topological black holes [18]. Of course, we should mention here that if one of spatial coordinates is compacted for the Ricci flat AdS black holes, then the AdS space-time in Poincare coordinates is no longer a ground state with respect to the black hole solutions, instead the so-called AdS soliton has more low energy and then there is a Hawking-Page phase transition between the Ricci flat black hole and AdS soliton [20-22]. The Ricci flat black holes mean zero curvature horizon black holes.

On the other hand, since people discovered the phenomenon of accelerated expansion of the universe at the end of last century, there have been a lot of proposals to explain the cause of this phenomenon. One way is so-called phantom dark energy with state equation, $p=\omega \rho$, where the state parameter satisfies $\omega<-1$, and $p$ and $\rho$ represent the pressure and energy density of phantom dark energy, respectively. To realize phantom dark energy, one can alter 
the mark in front of the kinetic term of a scalar field [23]. This is called phantom scalar field. Except for various studies in cosmology, the influence of phantom scalar field in black hole physics has also been extensively investigated. For instance, the destiny of a black hole owing to accretion of phantom dark energy has been studied in [24]. Thermodynamics of spherically symmetric black holes and critical phenomenon with phantom Maxwell field and phantom scalar field have been discussed in [25]. Very recently, people have studied thermodynamical geometry of AdS charged black holes with spherical horizon in [26].

In this study, we consider Einstein gravity theory with a negative cosmological constant and massless phantom scalar field. The black hole will have a Ricci flat horizon and phantom scalar fields only linearly depend on transverse coordinates. The thermodynamics of AdS phantom black hole are studied, and we find that although its horizon is Ricci flat and infinitely extended, the thermodynamical properties of the black hole and AdS Schwarzschild spherically symmetric black hole are qualitatively the same. In particular, HawkingPage phase transition can emerge between phantom black hole and the thermal gas solution in AdS space-time in Poincare coordinates. Further, we will calculate holographic entanglement entropy of a strip geometry which is dual to the AdS phantom black hole boundary following the proposal made by Ryu and Takayanagi in [27]. In recent papers on holographic superconductor models, it has been found that entanglement entropy plays a good probe role in revealing phase structure and phase transition in those systems. It can indicate the appearance of a new phase and gives the order of phase transition based on the behavior of entanglement entropy [28-33]. Very recently holographic entanglement entropy dual to a spherically symmetric AdS charged black hole has been calculated by Johnson [34]. It was found that the entanglement entropy can characterize the related phase transition for a charged AdS black hole. Further studies on holographic entanglement entropy and its relation to phase transition have been done for various AdS black holes in [3538]. For the AdS phantom black hole presented in this paper, we will further show such a relation which keeps valid even for the case with the Ricci flat horizon black hole.

\section{Phantom AdS Black Hole Solution}

We first consider a Einstein-Hilbert action with phantom massless scalar fields and a negative cosmological constant as

$$
S=\frac{1}{16 \pi G} \int d^{4} x \sqrt{-g}\left[R-2 \Lambda+\frac{1}{2} \sum_{i=1}^{2}\left(\partial \phi_{i}\right)^{2}\right] .
$$

In the above formula, $G$ is the Newtonian gravitational constant and $\phi_{i}$ stands for massless phantom scalar fields. In four dimensions, the relationship between the negative cosmological constant $\Lambda$ and the AdS curvature radius $l$ can be as follows:

$$
\Lambda=-\frac{3}{l^{2}}
$$

The Einstein's field equations corresponding with action (1) read

$$
R_{\mu \nu}-\frac{1}{2} g^{\mu \nu} R+\Lambda g^{\mu \nu}=T_{\mu \nu}
$$

where the energy momentum tensor is

$$
T_{\mu \nu}=\sum_{i=1}^{2}\left[-\nabla_{\mu} \phi_{i} \nabla_{\nu} \phi_{i}+\frac{1}{2} g_{\mu \nu}\left(\nabla \phi_{i}\right)^{2}\right]
$$

The motion equation of the phantom scalar field is

$$
\nabla^{2} \phi_{i}=0
$$

Now we consider a black hole solution which has a Ricci flat horizon in this system with the metric ansatz as

$$
d s^{2}=-f(r) d t^{2}+\frac{1}{f(r)} d r^{2}+r^{2}\left(d x^{2}+d y^{2}\right) .
$$

With this metric, one has the nonzero part of Ricci tensor as follows [18]:

$$
\begin{aligned}
& R_{t t}=\frac{1}{2} f f^{\prime \prime}+\frac{1}{r} f f^{\prime}, \\
& R_{r r}=-\frac{1}{2} \frac{f^{\prime \prime}}{f}-\frac{1}{r} \frac{f^{\prime}}{f}, \\
& R_{x x}=R_{y y}=-\left(f+r f^{\prime}\right),
\end{aligned}
$$

where $f^{\prime}=d f / d r$ and $f^{\prime \prime}=d^{2} f / d^{2} r$. The Ricci scalar can be written as follows:

$$
R=g^{t t} R_{t t}+g^{r r} R_{r r}+g^{i j} R_{i j}=-f^{\prime \prime}-\frac{4}{r} f^{\prime}-\frac{2 f}{r^{2}} .
$$

We take the scalar fields to be functions of transverse coordinates as follows:

$$
\begin{aligned}
& \phi_{1}=\alpha x, \\
& \phi_{2}=\alpha y,
\end{aligned}
$$

where $\alpha$ is a constant; then it is found that the equations of motions for both gravitational field and matter field are satisfied if $f(r)$ takes the following form:

$$
f(r)=\alpha^{2}+\frac{r^{2}}{l^{2}}-\frac{2 M}{r},
$$

in which $M$ is a parameter relating to the mass of the solution. In fact, this solution describes an AdS black hole with a Ricci flat horizon. As we can see that the influence of the phantom scalar in the solution is manifested by parameter $\alpha$. In addition, note that the scalar field enters the action only through its derivative and therefore enjoys a shift symmetry. These scalar fields are only determined up to a constant. When the phantom scalar fields are absent, the solution reduces to the AdS black hole solution with Ricci flat horizon in Einstein gravity. In that case, one has $\alpha=0$. A natural generalization of solution (10) is to include electric and 
magnetic charge from Maxwell field. Namely if a Maxwell field appears in action (1), then one has a dynamic phantom AdS black hole solution as

$$
f(r)=\alpha^{2}+\frac{r^{2}}{l^{2}}-\frac{2 M}{r}+\frac{e^{2}+g^{2}}{r^{2}},
$$

where $e$ and $g$ are electric charge and magnetic charge of the solution, respectively. Here we mention that one can easily generalize these black hole solutions (10) and (11) to higher dimensional $(d>4)$ cases. Finally we should stress that if the scalar fields are the usual canonical ones in (1), namely, the sign of the kinetic term in the scalar fields formula is negative, this AdS black hole solution was first found in [39] with the metric function:

$$
f(r)=-\alpha^{2}+\frac{r^{2}}{l^{2}}-\frac{2 M}{r} .
$$

In that case, the thermodynamical properties of solution (12) and the topological black hole with a horizon of negative constant curvature space are qualitatively the same in AdS space-time [18].

\section{Thermodynamics of the Phantom AdS Black Hole}

In this section, the thermodynamics of this AdS black hole solution are discussed. Here we are discussing the case where the transverse coordinates $x$ and $y$ are infinitely extended. In other words, the directions spanned by $x$ and $y$ are not compacted. In that case, precisely speaking, what we are discussing is not a black hole, but a black brane. And the vacuum of the system is no longer the AdS solution, but an AdS space in Poincare coordinates with metric function:

$$
f(r)=\frac{r^{2}}{l^{2}} .
$$

On the other hand, let us note that when $\alpha^{2}=1$, the metric function in (10) is nothing, but the one for a Schwarzschild AdS black hole is with a spherical horizon. Thus one may expect that the thermodynamic properties of the black hole solution (10) are essentially the same as those of AdS Schwarzschild black holes. Here we will show this indeed holds. To make qualitative comparison, we take that the area spanned by $x$ and $y$ is $4 \pi$. So parameter $M$ in (10) is just the mass of the solution with respect to the AdS vacuum in Poincare coordinates. In this paper we take the units with $G=c=h=k_{b}=1$.

The horizon of the black hole is given by $\left.f(r)\right|_{r=r_{+}}=0$. Thus the black hole mass can be expressed in terms of the horizon radius $r_{+}$as

$$
M=\frac{\alpha^{2}}{2} r_{+}+\frac{r_{+}^{3}}{2 l^{2}} .
$$

As a function of the horizon radius $r_{+}, M$ is a monotonically increasing function. In other words, this equation shows that for any positive value of $M$ there is a corresponding horizon.
And only when the case $r_{+} \rightarrow 0, M \rightarrow 0$. The Hawking temperature $T_{H}$ of the black hole can be obtained in several ways. One way is that the Euclidean time of the black hole solution should take a period so that the potential conical singularity can be avoided at the horizon of the black hole and the inverse of the period just gives the Hawking temperature. In this way, one has the Hawking temperature of the black hole as

$$
T=\frac{1}{4 \pi r_{+}}\left(\alpha^{2}+\frac{3 r_{+}^{2}}{l^{2}}\right) .
$$

In Einstein's gravity theory, the entropy of black hole obeys the well-known Bekenstein-Hawking area formula. In our case, the entropy of black hole can be expressed as follows:

$$
S=\pi r_{+}^{2}
$$

Substituting (16) into (15) and eliminating $r_{+}, T$ is given by

$$
T=\frac{\alpha^{2}+3 S / \pi l^{2}}{4 \sqrt{\pi} \sqrt{S}} .
$$

It is easy to show that the mass, entropy, and the temperature of the black hole obey the first law of black hole thermodynamics:

$$
d M=T d S
$$

To study the thermodynamical stability of the black hole, let us calculate the heat capacity:

$$
C=T \frac{\partial S}{\partial T}=\frac{\partial M}{\partial T}=\frac{2 l^{2} \pi r_{+}^{2}\left(3 r_{+}^{2} / l^{2}+\alpha^{2}\right)}{3 r_{+}^{2}-l^{2} \alpha^{2}}
$$

We can see that the heat capacity is positive when $r_{+}>l \alpha / \sqrt{3}$, negative when $r_{+}<l \alpha / \sqrt{3}$, and divergent when $r_{+}=l \alpha / \sqrt{3}$. This means that the black hole is locally thermodynamically unstable for small horizons with $r_{+}<l \alpha / \sqrt{3}$, while it is locally thermodynamically stable for large horizons with $r_{+}>$ $l \alpha / \sqrt{3}$. Figure 1 shows the temperature-entropy relation. And it is easy to see that the small entropy is thermodynamically unstable, while the large entropy is thermodynamically stable.

The Hawking temperature (15) can be reexpressed as

$$
\frac{3}{l^{2}} r_{+}^{2}-4 \pi k_{B} T r_{+}+\alpha^{2}=0
$$

Through this relation, we can see that there exists a minimal temperature as

$$
T_{\min }=\frac{\alpha \sqrt{3}}{2 \pi l} .
$$

In that case, corresponding horizon radius is given by

$$
r_{0}=\frac{\alpha l}{\sqrt{3}}
$$



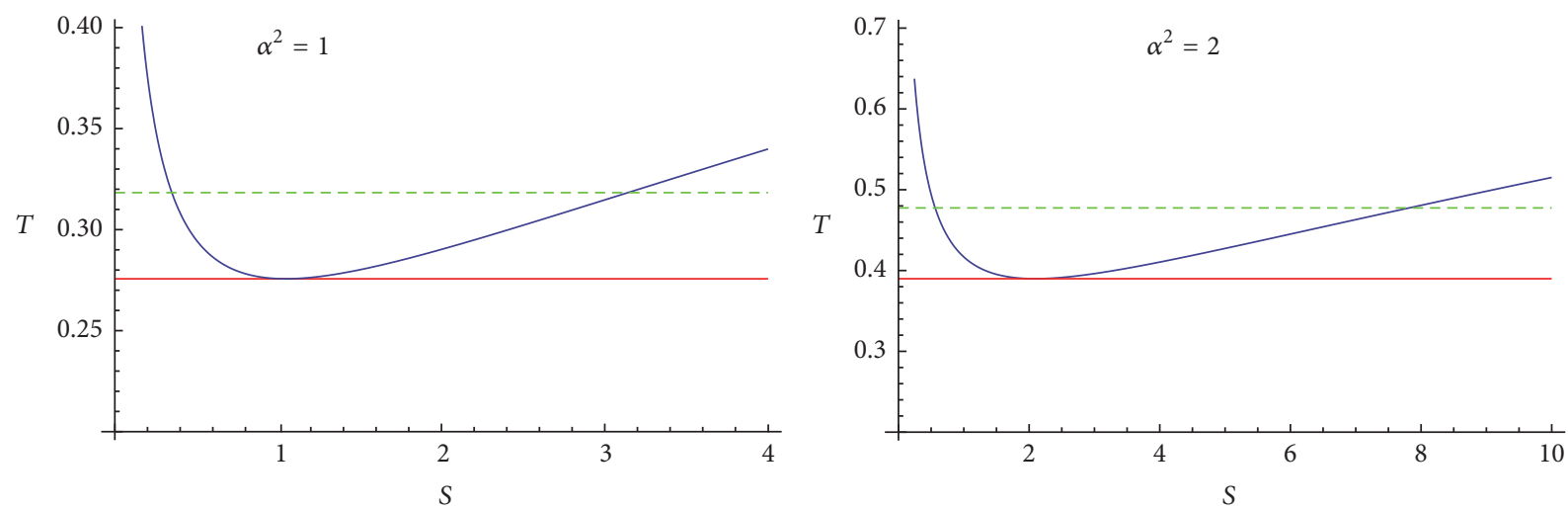

FIGURE 1: The black hole temperature in terms of entropy. The dashed green line represents the Hawking-Page transition temperature, while the solid red line stands for $T_{\min }$. When $T<T_{\min }$, there is only a thermal gas solution in the AdS space. When $T>T_{\min }$, for a given temperature $T$, there exist two black hole solutions given by (23). The one with large horizon is stable, while it is thermodynamically unstable for the smaller one.
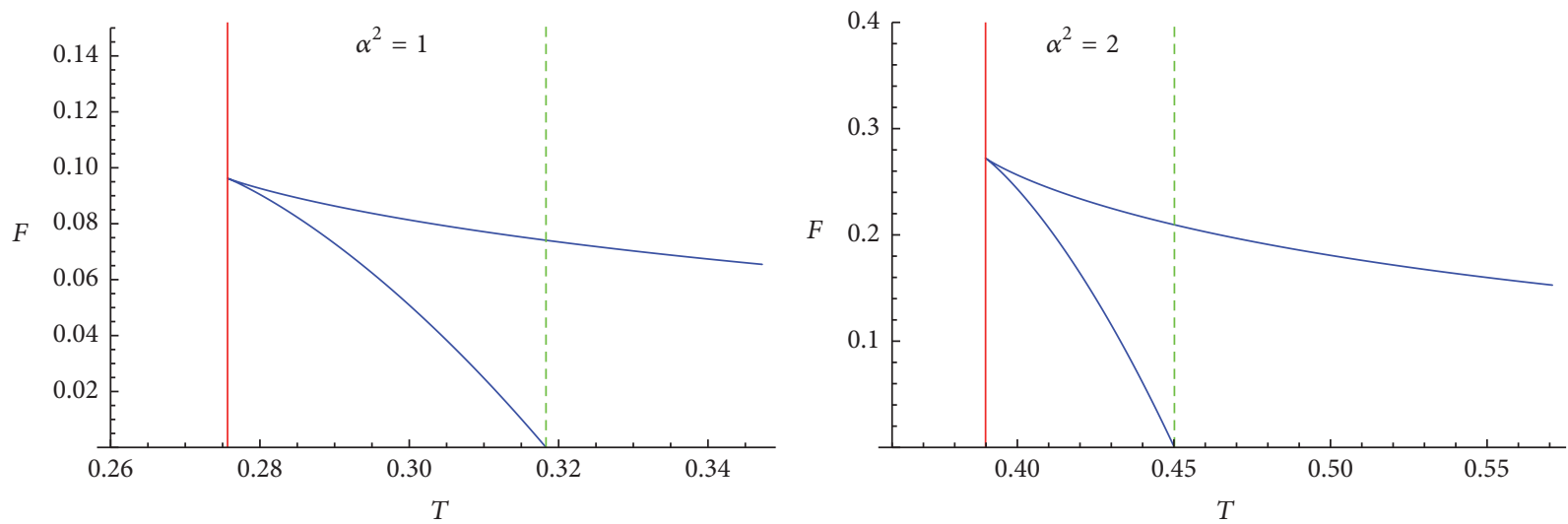

Figure 2: The Helmholtz free energy of the black hole as a function of temperature T. The dashed green line stands for the Hawking-Page transition temperature, while the solid red line represents the minimal temperature.

When $T>T_{\min }$, for a given temperature, there are two black hole horizons:

$$
r_{l, s}=\frac{\alpha^{2} T}{2 \pi T_{\min }^{2}}\left(1+\sqrt{1-\frac{T_{\min }^{2}}{T^{2}}}\right) .
$$

The small black hole with negative heat capacity is thermodynamically unstable, while the larger one with positive heat capacity is thermodynamically stable.

With the mass, entropy, and temperature, the Helmholtz free energy is easily calculated as

$$
F=M-T S=\frac{1}{4} r_{+}\left(-\frac{r_{+}^{2}}{l^{2}}+\alpha^{2}\right) .
$$

According to the above, we can easily see that the free energy is equivalent to the Euclidean action by setting the pure AdS vacuum in Poincare coordinates as a ground state. We can see from the free energy that the free energy is positive when $r_{+}<l \alpha$, while it is negative when $r_{+}>l \alpha$. It implies that the black hole is globally thermodynamically unstable when $r_{+}<l \alpha$, while it is globally thermodynamically stable when $r_{+}>l \alpha$. Namely, there is a Hawking-Page phase transition when the free energy vanishes and $r_{+}=l \alpha$. The HawkingPage transition temperature is given by

$$
T_{\mathrm{HP}}=\frac{\alpha}{\pi l} \text {. }
$$

When $T>T_{\mathrm{HP}}$, the system is dominated by the black hole solution; while it is dominated by a thermal gas solution as $T<T_{\mathrm{HP}}$. When $T=T_{\mathrm{HP}}$, the Hawking-Page transition happens between the black hole phase and thermal gas phase.

From (21) and (25), we can see that the phantom scalar field has some effect on the thermodynamic stability and the Hawking-Page transition temperature through the parameter $\alpha$. But the ratio between these two temperatures,

$$
D=\frac{T_{\mathrm{HP}}}{T_{\min }}=\frac{2 \sqrt{3}}{3},
$$

is independent of the parameter $\alpha$. The ratio is the same as the one for the case of the AdS Schwarzschild black hole. We show the free energy of the black hole as a function of temperature in Figure 2. 


\section{Entanglement Entropy and Hawking-Page Transition}

For a quantum mechanical system with many degrees of freedom, we separate the total system into two subsystems $A$ and $B$. The Hilbert space of the total system can be expressed by the direct product of the two subsystems $A$ and $B$ as $H_{\text {total }}=H_{A} \otimes H_{B}$. For the observer who is only accessible to subsystem $A$, because the information of $B$ cannot be directly observed, the total system is described by reduced density matrix $\rho_{A}$ :

$$
\rho_{A}=\operatorname{tr}_{B} \rho_{\text {total }},
$$

where the trace is taken only over the Hilbert space of subsystem $B$. The entanglement entropy of the subsystem $A$ is defined as

$$
S_{A}=-\operatorname{tr}_{A} \rho_{A} \log \rho_{A} .
$$

Usually it is very difficult to calculate entanglement entropy in quantum field theory. However, the AdS/CFT correspondence provides a powerful tool to calculate the entanglement entropy for a strong coupling conformal field theory in a simple way. Suppose that the boundary of the subsystem $A$ is $\partial A$ in the boundary of the AdS space-time. The entanglement entropy of the subsystem $A$ is given by the following formula [27]:

$$
S_{A}=\frac{\operatorname{Area}\left(\gamma_{A}\right)}{4 G}
$$

where $G$ is the Newton gravitational constant in the AdS bulk and $\gamma$ is the minimal surface extended into the bulk with boundary $\partial \gamma=\partial A$.

Now we will take formula (29) to calculate the entanglement entropy of the conformal field theory dual to the AdS black hole solution given by (10). To study the entanglement entropy, we should choose a proper region for $A$. Here we consider a rectangular strip parameterized by the boundary coordinates $x$ and $y$, and we assume $y$-direction is infinitely extended and direction $x$ has width $\ell$. Thus we can use $x$ to parameterize the minimal surface.

With the help of (6) and (29), we can obtain the area of the minimal surface for the strip geometry as follows:

$$
\operatorname{Area}\left(\gamma_{A}\right)=L \int_{-\ell / 2}^{\ell / 2} d x \sqrt{\frac{\left(r^{\prime}\right)^{2}}{f(r)}+r} \text {, }
$$

where $r^{\prime}=d r / d x$ and $L$ is the length along $y$-direction, which will be set to be unity in what follows. Note that the integrand in (30) does not depend explicitly on $x$. We can derive the equation of motion of $r(x)$ as

$$
\begin{aligned}
4 r(x)^{2} f(r)^{2}-2 f(r) r^{\prime}(x)^{2}+r(x) r^{\prime}(x)^{2} f^{\prime}(r) \\
-2 f(r) r r^{\prime \prime}(x)=0 .
\end{aligned}
$$

Due to the symmetry of the minimal surface, obviously we have

$$
\begin{aligned}
r(0) & =r_{0}, \\
r^{\prime}(0) & =0,
\end{aligned}
$$

at the returning point $r=r_{0}$ where $x=0$. With condition (32), we can solve (31) numerically and obtain function $r(x)$. Then substituting $r(x)$ into (30), we can get the entanglement entropy. It is found that the entanglement entropy is always divergent. In order to get more meaningful physically results, we introduce an ultraviolet cut-off. Here we are interested in the regularized entropy $\delta S=S-S_{0}$, where $S_{0}$ is the entanglement entropy for the same geometry, but the bulk is the pure AdS space in Poincare coordinates, which is also calculated with numerical method. In Figure 3, as typical examples, we plot the regularized entanglement entropy versus the temperature of the black hole in the cases $\ell=0.6$, $\ell=1.2$ and $\alpha^{2}=1, \alpha^{2}=2$, the corresponding ultraviolet cut-off is set to be $r(0.59), r(1.19)$. Comparing Figure 3 with Figure 1, we find that the behavior of the entanglement entropy is quite similar to that of the black hole entropy, and the corresponding minimal temperatures $T_{\min }$, which is shown by solid red line in Figure 3, are the same as those in Figure 1.

In Figure 3, we also show the Hawking-Page transition temperature with dashed green line. With (26), it shows that the ratio between the minimal temperature and HawkingPage transition temperature also keeps valid in the behavior of the entanglement entropy. In addition, we notice that, with the change of the width $\ell$, the entanglement entropy changes consequently, but the phase structure of the entanglement entropy does not changes and the minimal temperature does not change. We can therefore conclude that the entanglement entropy can indeed reveal the phase structure of the AdS phantom black hole.

\section{Discussion and Conclusions}

Black hole physics is one of important topics in general relativity and quantum gravity. Thermodynamics, quantum mechanics, statistical physics, and information theory are entangled with each other in black hole thermodynamics. It is generally believed that to completely understand black hole thermodynamics will be greatly helpful to establish a self-consistent quantum gravity theory. In this respect, the AdS black hole might play a central role since the AdS/CFT correspondence relates a quantum gravity in AdS spacetime to a conformal field theory in the AdS boundary. In this paper, we have presented an AdS black hole solution with Ricci flat horizon in Einstein-phantom scalar theory. The phantom scalar fields only depend on the transverse coordinates $x$ and $y$ and are parameterized by the parameter $\alpha$ (see (9)). However, we noticed that the parameter $\alpha$ can be renormalized to be one by the following rescaling:

$$
\begin{gathered}
t \longrightarrow \frac{t}{\alpha}, \\
r \longrightarrow \alpha r, \\
(x, y) \longrightarrow \frac{(x, y)}{\alpha}, \\
M \longrightarrow \alpha^{3} M .
\end{gathered}
$$



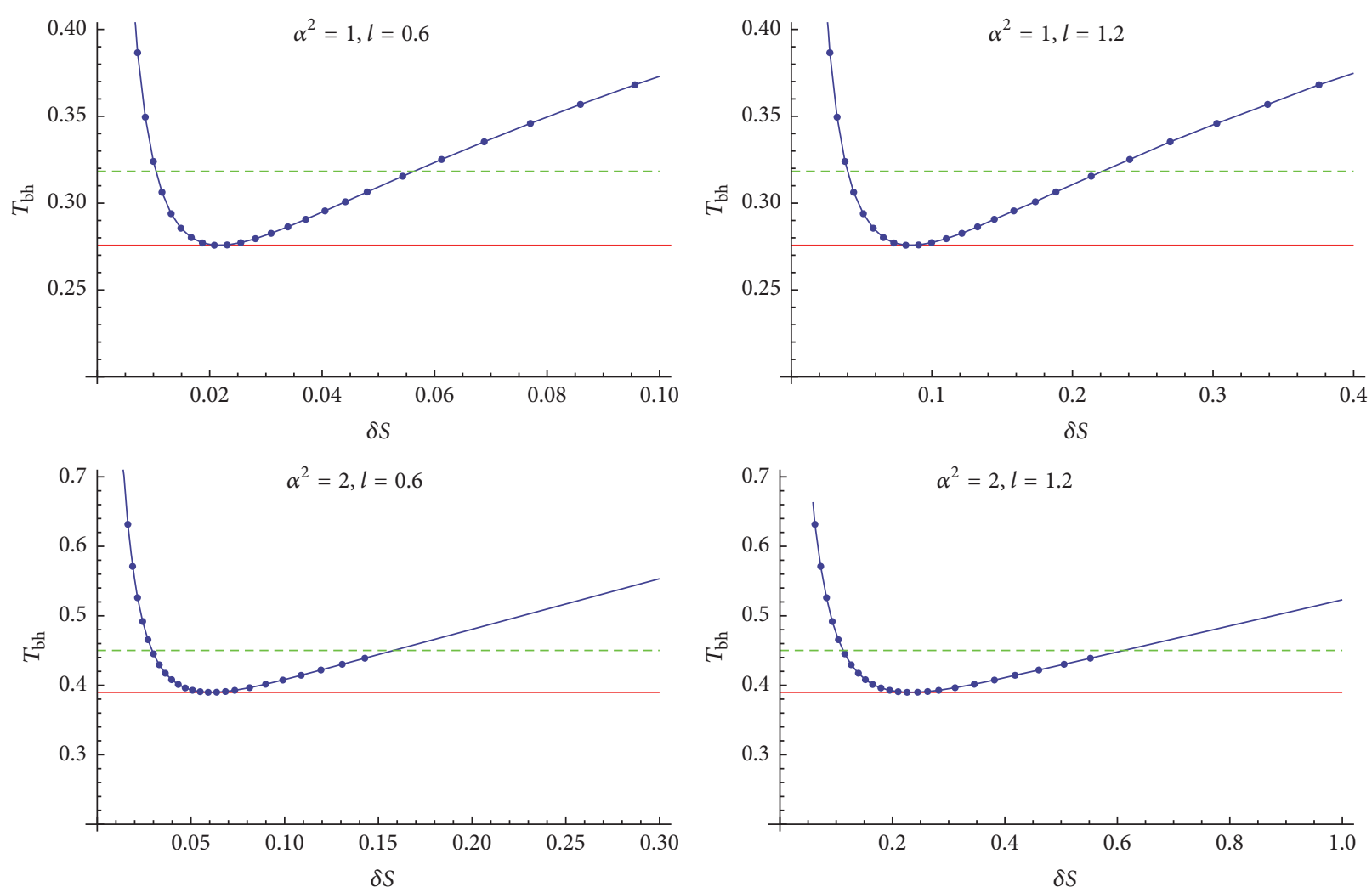

FiguRE 3: The regularized entanglement entropy versus the black hole temperature. The dashed green line represents the Hawking-Page transition temperature, while the solid red line stands for the minimal temperature.

With the rescaling (33), the black hole solution can be rewritten as

$$
d s^{2}=-g(r) d t^{2}+g^{-1}(r) d r^{2}+r^{2}\left(d x^{2}+d y^{2}\right),
$$

with

$$
g(r)=1+\frac{r^{2}}{l^{2}}-\frac{2 M}{r} .
$$

Comparing this black hole solution with the AdS Schwarzschild black hole,

$$
\begin{aligned}
d s^{2}= & -g(r) d t^{2}+g^{-1}(r) d r^{2} \\
& +r^{2}\left(d \theta^{2}+\sin ^{2} \theta d \varphi^{2}\right),
\end{aligned}
$$

we note that the only difference is the replacement of the 2dimensional Euclidean space $R^{2}$ by the 2-dimensional sphere $S^{2}$.

Indeed we have found that although its horizon structure is Ricci flat, the AdS black hole with phantom scalar has qualitatively same thermodynamic properties as those of AdS Schwarzschild black hole: there exists a minimal temperature and small black hole is thermodynamically unstable, while large black hole is thermodynamically stable. And in particular, the Hawking-Page phase transition can happen between the AdS phantom black hole and the thermal gas solution in AdS space-time in Poincare coordinate.
Further we have calculated the entanglement entropy of dual conformal field theory for a strip geometry in the AdS boundary by the Ryu-Takayanagi proposal. It was found that the behavior of the entanglement entropy is also qualitatively the same as that of the thermodynamical entropy of the black hole. This example further supports that the black hole entropy might indeed be interpreted as entanglement entropy. On the other hand, it also indicates entanglement entropy is indeed a good probe to phase structure and phase transition in black hole thermodynamics.

\section{Conflicts of Interest}

The authors declare that they have no conflicts of interest.

\section{Acknowledgments}

This work is supported by Meritocracy Research Funds (463508) of China West Normal University and the authors thank R. G. Cai for helpful suggestions and discussions.

\section{References}

[1] S. W. Hawking and D. N. Page, "Thermodynamics of black holes in anti-de Sitter space," Communications in Mathematical Physics, vol. 87, no. 4, pp. 577-588, 1983.

[2] J. Maldacena, "The large- $N$ limit of superconformal field theories and supergravity," International Journal of Theoretical Physics, vol. 38, no. 4, pp. 1113-1133, 1999. 
[3] S. S. Gubser, I. R. Klebanov, and A. M. Polyakov, "Gauge theory correlators from non-critical string theory," Physics Letters B, vol. 428, no. 1-2, pp. 105-114, 1998.

[4] E. Witten, "Anti de Sitter space and holography," Advances in Theoretical and Mathematical Physics, vol. 2, no. 2, pp. 253-291, 1998.

[5] E. Witten, "Anti-de Sitter space, thermal phase transition, and confinement in gauge theories," Advances in Theoretical and Mathematical Physics, vol. 2, no. 3, pp. 505-532, 1998.

[6] J. P. Lemos, "Two-dimensional black holes and planar general relativity," Classical and Quantum Gravity, vol. 12, no. 4, pp. 1081-1086, 1995.

[7] J. P. S. Lemos, "Cylindrical black hole in general relativity," Physics Letters B, vol. 353, pp. 46-51, 1994.

[8] C. G. Huang and C. B. Liang, "A torus-like black hole," Physics Letters A, vol. 201, no. 1, pp. 27-32, 1995.

[9] J. P. Lemos and V. T. Zanchin, "Rotating charged black strings and three-dimensional black holes," Physical Review D, vol. 54, no. 6, pp. 3840-3853, 1996.

[10] R.-G. Cai and Y.-Z. Zhang, "Black plane solutions in fourdimensional spacetimes," Physical Review D, vol. 54, no. 8, pp. 4891-4898, 1996.

[11] R.-G. Cai, J.-Y. Ji, and K.-S. Soh, “Topological dilaton black holes," Physical Review D, vol. 57, no. 10, pp. 6547-6550, 1998.

[12] S. Åminneborg, I. Bengtsson, S. Holst, and P. Peldán, "Making anti-de Sitter black holes," Classical and Quantum Gravity, vol. 13, no. 10, pp. 2707-2714, 1996.

[13] R. B. Mann, "Pair production of topological anti-de Sitter black holes," Classical and Quantum Gravity, vol. 14, no. 5, pp. L109L114, 1997.

[14] L. Vanzo, "Black holes with unusual topology," Physical Review D, vol. 56, no. 10, pp. 6475-6483, 1997.

[15] D. R. Brill, J. Louko, and P. Peldán, “Thermodynamics of (3+ 1)-dimensional black holes with toroidal or higher genus horizons," Physical Review D, vol. 56, no. 6, pp. 3600-3610, 1997.

[16] D. Klemm, V. Moretti, and L. Vanzo, "Rotating topological black holes," Physical REView D, vol. 57, pp. 6127-6137, 1998.

[17] R.-G. Cai and K.-S. Soh, "Topological black holes in the dimensionally continued gravity," Physical Review D, vol. 59, no. 4, Article ID 044013, 1999.

[18] D. Birmingham, "Topological black holes in anti-de Sitter space," Classical and Quantum Gravity, vol. 16, no. 4, pp. 11971205, 1999.

[19] R.-G. Cai, "Gauss-Bonnet black holes in AdS spaces," Physical Review D, vol. 65, Article ID 084014, 2002.

[20] S. Surya, K. Schleich, and D. M. Witt, "Phase transitions for flat AdS black holes," Physical Review Letters, vol. 86, no. 23, pp. 5231-5234, 2001.

[21] R.-G. Cai, S. P. Kim, and B. Wang, "Ricci flat black holes and Hawking-Page phase transition in Gauss-Bonnet gravity and dilaton gravity," Physical Review D, vol. 76, no. 2, Article ID 024011, 2007.

[22] A. Anabalón, D. Astefanesei, and D. Choque, "Hairy AdS solitons," Physics Letters B, vol. 762, pp. 80-85, 2016.

[23] R. R. Caldwell, "A phantom menace? Cosmological consequences of a dark energy component with super-negative equation of state," Physics Letters B, vol. 545, no. 1-2, pp. 23-29, 2002.

[24] E. Babichev, V. Dokuchaev, and Y. Eroshenko, "Black hole mass decreasing due to phantom energy accretion," Physical Review Letters, vol. 93, no. 2, Article ID 021102, 2004.
[25] M. Azreg-Aïnou, G. T. Marques, and M. E. Rodrigues, "Phantom black holes and critical phenomena," Journal of Cosmology and Astroparticle Physics, vol. 2014, no. 7, article 36, 2014.

[26] H. Quevedo, M. N. Quevedo, and A. Sánchez, "Geometrothermodynamics of phantom AdS black holes," European Physical Journal C, vol. 76, no. 3, article 110, 2016.

[27] S. Ryu and T. Takayanagi, "Holographic derivation of entanglement entropy from AdS/CFT", Physical Review Letters, vol. 96, Article ID 181602, 2006.

[28] T. Albash and C. V. Johnson, "Holographic studies of entanglement entropy in superconductors," Journal of High Energy Physics, vol. 2012, no. 5, article 79, pp. 1-21, 2012.

[29] R.-G. Cai, S. He, L. Li, and Y.-L. Zhang, "Holographic entanglement entropy in P-wave superconductor phase transition," Journal of High Energy Physics, vol. 2012, no. 7, article 27, 2012.

[30] R. G. Cai, S. He, L. Li, and L. F. Li, "Entanglement entropy and Wilson loop in Stückelberg holographic insulator/superconductor model," Journal of High Energy Physics, vol. 2012, no. 10, article 107, 2012.

[31] R. G. Cai, L. Li, L. F. Li, and R. K. Su, "Entanglement entropy in holographic P-wave superconductor/insulator model," Journal of High Energy Physics, vol. 2013, article 63, 2013.

[32] R.-G. Cai, S. He, L. Li, and Y.-L. Zhang, "Holographic entanglement entropy in insulator/superconductor transition," Journal of High Energy Physics, vol. 2012, no. 7, article 88, 2012.

[33] L.-F. Li, R.-G. Cai, L. Li, and C. Shen, "Entanglement entropy in a holographic p-wave superconductor model," Nuclear Physics $B$, vol. 894, pp. 15-28, 2015.

[34] C. V. Johnson, "Large $N$ phase transitions, finite volume, and entanglement entropy," Journal of High Energy Physics, vol. 2014, article 47, 2014.

[35] X. X. Zeng, H. Zhang, and L. F. Li, "Phase transition of holographic entanglement entropy in massive gravity," Physics Letters B, vol. 756, pp. 170-179, 2016.

[36] X.-X. Zeng, X.-M. Liu, and L.-F. Li, "Phase structure of the Born-Infeld-anti-de Sitter black holes probed by non-local observables," European Physical Journal C, vol. 76, no. 11, article 616, 2016.

[37] X. X. Zeng and L. F. Li, "Van der waals phase transition in the framework of holography," Physics Letters B, vol. 764, pp. 100108, 2017.

[38] A. Dey, S. Mahapatra, and T. Sarkar, "Thermodynamics and entanglement entropy with Weyl corrections," Physical Review D, vol. 94, no. 4, Article ID 026006, 2016.

[39] Y. Bardoux, M. M. Caldarelli, and C. Charmousis, "Shaping black holes with free fields," Journal of High Energy Physics, vol. 2012, article 54, 2012. 

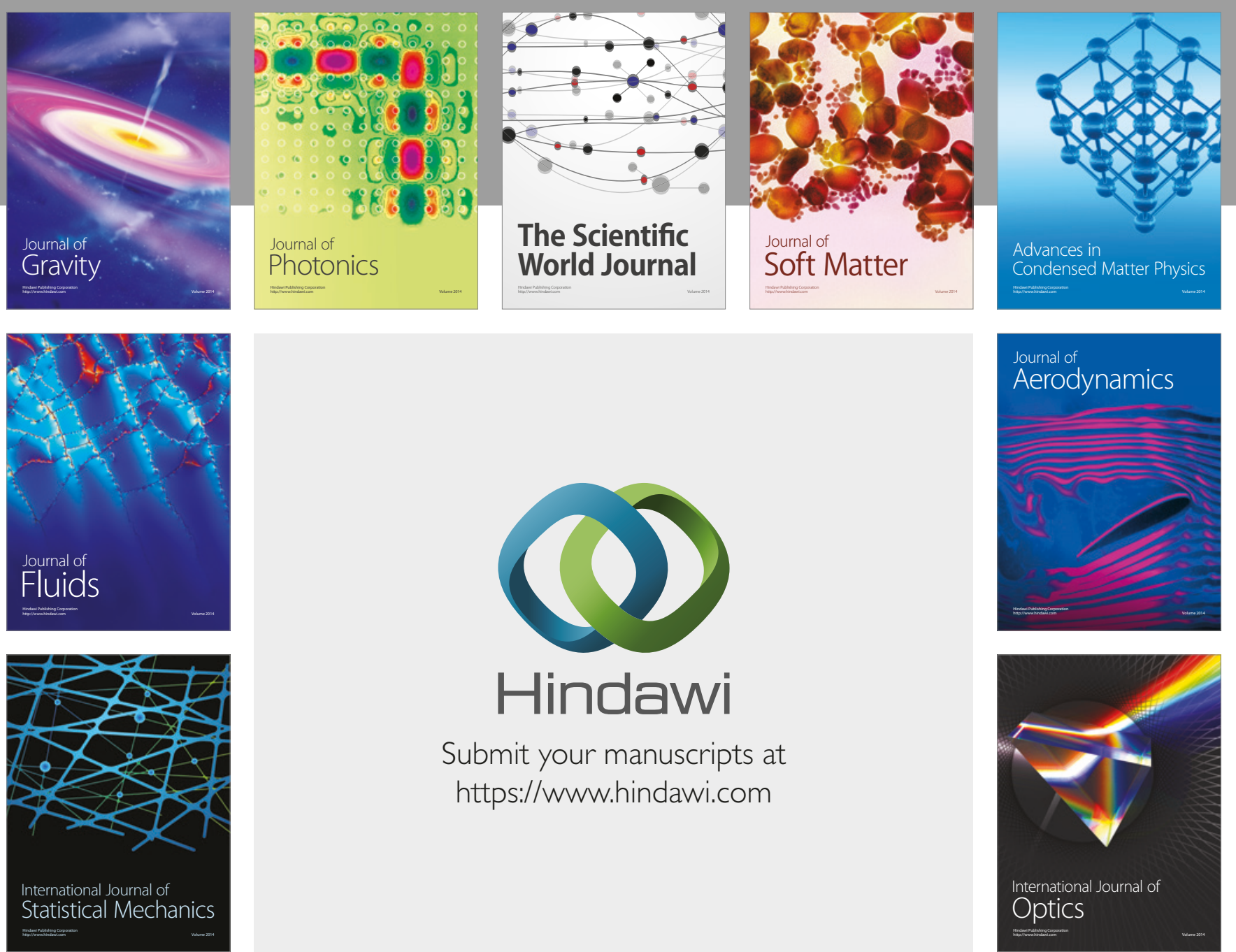

Submit your manuscripts at

https://www.hindawi.com
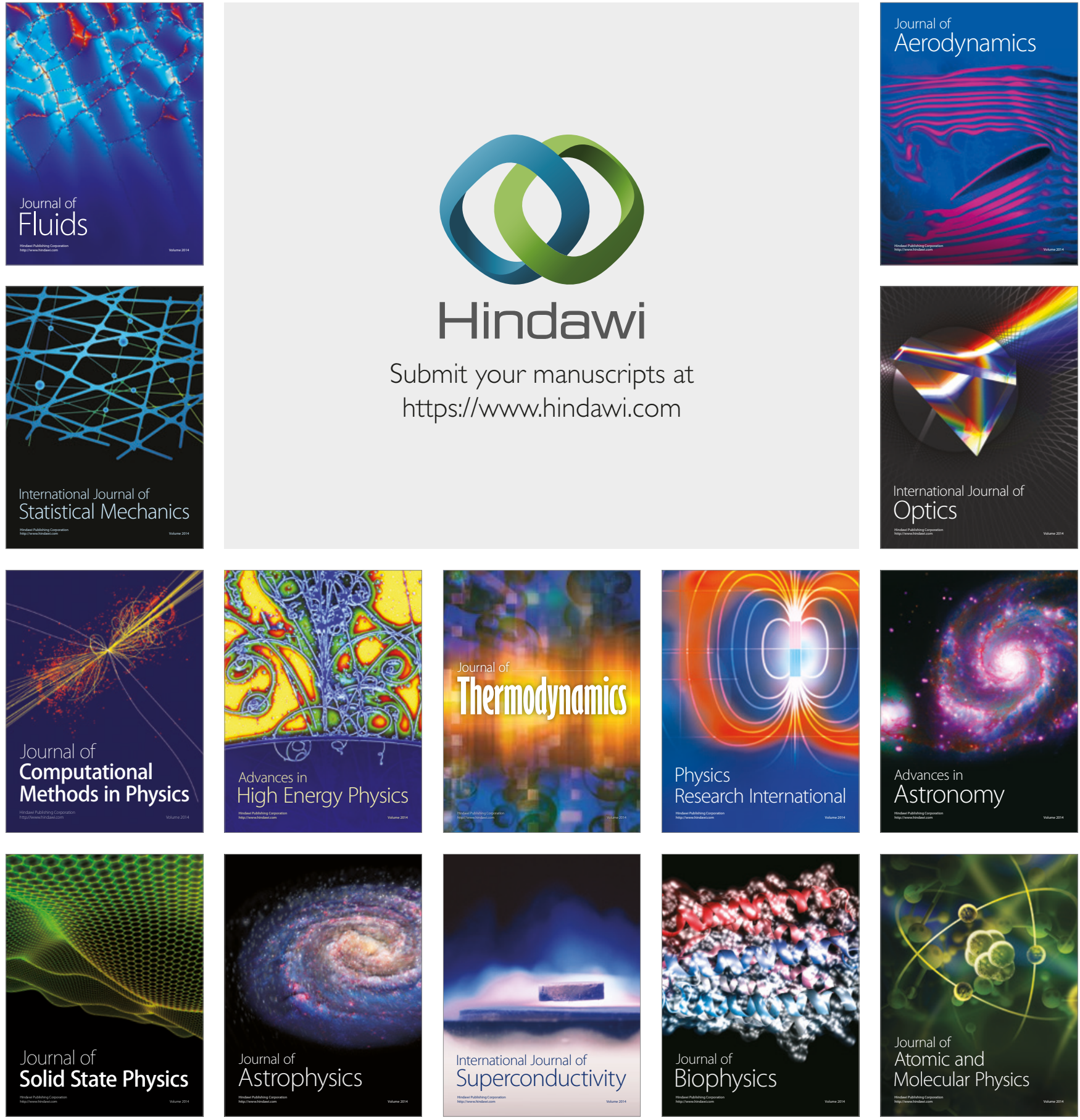